\title{
Über die quantitative Bestimmung der Aminosäuren, Polypeptide und der Hippursäure im Harne durch Formoltitration.

\author{
von
} \\ V. Henriques und S. P. L. Sörensen.
}

(Aus dem physiologischen Laboratorium der kgl. tierärztlichen und landwirtschaftlichen Hochschule und aus dem Carlsberg-Laboratorium, Kopenhagen.)

(Der Redaktion zugegangen am 24. September 1909.)

In einem der letzten Hefte dieser Zeitschrift ${ }^{1}$ ) hat $\mathrm{H}$. Malfatti eine Abhandlung: «Die Formoltitration der Aminosäuren im Harne veröffentlicht und darin auch die von uns für die Aminosäurebestimmung im Harne ausgearbeitete Methode ${ }^{2}$ ) eingehend besprochen. Malfatti schreibt (l. c. S. 503): *Nun erscheint allerdings die von Henriques angewandte Methode nicht einwandsfrei; es geht nicht an, den auf Lackmusneutralität gebrachten Harn mit Phenolphthalein weiter zu titrieren, sonst wird die ganze Laugenmenge, welche der lackmusneutrale Harn braucht, um auch ohne Formolzusatz phenolphtaleinneutral zu werden, fälschlich den Ergebnissen der Formoltitration zugemessen.»

Es erhellt hieraus, daß selbst ein so geschickter und in formoltitrimetrischen Arbeiten geübter Forscher wie Herr Malfatti nicht mit allen Einzelheiten des Prinzipes der Methode vertraut ist, und wir haben es daher für wünschenswert gehalten, die prinzipielle Grundlage der Methode etwas ausführlicher klarzulegen, als es in der eben zitierten Abhandlung von V. Henriques geschehen ist.

Die formoltitrimetrische Bestimmung der Stickstoffmenge, die als Ammoniak oder primäre Aminoverbindungen, z. B. die

1) Diese Zeitschrift, Bd. LXI, S. 499 (1909).

2) Diese Zeitschrift, Bd. LX, S. 2 (1909). 
üblichen Aminosäuren, in einer Lösung vorhanden ist, zerfällt in $z$ wei Teile:

Erstens die Neutralisation der Lösung, womit bezweckt wird, die Lösung in dem Sinne neutral zu machen, daß gleich viele saure und basische Gruppen anwesend sind, und zweitens die eigentliche Formoltitrierung, wodurch die Ammoniak- und Aminogruppen durch Formol neutralisiert werden, sodaß eine äquivalente Säuremenge frei wird und titrimetrisch bestimmt werden kann. Es ist hier die Rede von zwei ganz verschiedenen titrimetrischen Operationen, von welchen jede eben den für das bezweckte Ziel geeigneten Indikator erfordert. Bei der Neutralisation der Lösung bedingen die anwesenden schwach basischen Ammoniak- und Aminogruppen die Anwendung eines Indikators, welcher bei der eigentlichen Formoltitrierung, wobei es sich um eine Neutralisation schwach saurer Gruppen handelt, oft ganz unbrauchbar sein wird. Im folgenden werden wir durch einige experimentelle Data klarlegen, daß die hier angeführten, auf einfachen theoretischen Schlußfolgerungen fußenden Betrachtungen mit den Versuchsergebnissen im Einklang stehen. Wir werden erst die eigentliche Formoltitrierung und dann die Neutralisation der Lösung besprechen.

Die eigentliche Formoltitrierung. In seiner Hauptabhandlung ${ }^{1}$ ) über die Formoltitrierung hat Sörensen stark hervorgehoben, daß die durch die Einwirkung von Formol auf eine Aminosäure, z. B. Alanin, sich vollziehende chemische Umsetzung :

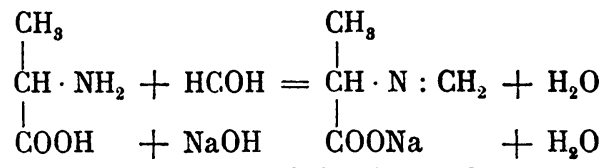

reziprok ist und daß der schließliche Gleichgewichtszustand nicht nur von der Formol- und Wassermenge, sondern auch von der Wasserstoffionenkonzentration der Lösung abhängig ist. Um die Formoltitrierung möglichst quantitativ durchführen, d.h. um eine möglichst vollständige Umsetzung von links nach

1) Biochemische Zeitschrift, Bd. VII, S. 45 (1907). 
rechts (siehe obige Gleichung) hervorrufen zu können, ist es demnach notwendig, nicht nur einen großen Überschuß an Formol und so wenig Wasser wie tunlichst anzuwenden, sondern auch einen Indikator zu benutzen, dessen Umschlag erst bei einer genügend großen Hydroxylionen- oder - wenn man es so ausdrücken will - einer genügend kleinen Wasserstoffionenkonzentration stattfindet. Lackmus (dessen Umschlag bei einer Wasserstoffionenkonzentration von ca. $10^{-7}$ erfolgt) ist daher als Indikator bei der Formoltitrierung ungeeignet, und auch bei Anwendung von Phenolphthalein erhält man gewöhnlich zu niedrige Resultate, wenn nur bis zum schwachen rosa Farbton (einer Wasserstoffionenkonzentration von $10^{-8,3}-10^{-8,4}$ entsprechend) titriert wird. Selbs . bei Titrierung bis zu deutlich roter Farbe (Wasserstoffionenkonzentration $10^{-8,7}-10^{-8,8}$ ) spielt diese Fehlerquelle bisweilen eine Rolle und erst bei Titrierung bis zu stark roter Farbe (Wasserstoffionenkonzentration: $\left.10^{-9,0}-10^{-9,1}\right)$ ist die Hydroxylionenkonzentration der Lösung so stark angewachsen, daß die öfters erwähnte Umsetzung so gut wie vollständig von links nach rechts verläuft; doch bekommt man, wie es deutlich aus den Resultaten Sörensens (1. c. S. 79) hervorgeht, auch in diesem Falle stets Minimalwerte (durchschnittlich $97,5 \%$ der berechneten Werte). In der eben zitierten Abhandlung Sörensens (1. c. S. 64) findet sich eine genaue Beschreibung über die - übrigens sehr einfache - Darstellung einer Kontrollösung, welche die gewünschte stark rote Farbe hat, und auf deren Farbstärke alle vorliegenden Analysen zu titrieren sind. Aus den in der Abhandlung tabellarisch zusammengestellten Versuchsergebnissen erhellt, daß die hier erwähnte Fehlerquelle bei der Titrierung von Ammoniumchlorid oder von Glykokoll nicht, bei der Titrierung von Alanin oder Leucin dagegen deutlich merkbar ist. Hiermit übereinstimmend findet Malfatti, der bis zu schwach roter Farbe titriert, nur beim Glykokoll befriedigende Resultate: «Bei Alanin wurden noch halbwegs befriedigende Werte erhalten, bei Valin, Leucin, Tyrosin und einem Aminosäurengemisch aus Eiweiß waren die Resultate nicht befriedigend* (l. c. S. 501). Da es nicht als ausgeschlossen betrachtet werden 
kann, daß derartige Aminosäuren wie die letzterwähnten im Harne vorkommen können, und da die bei der Formoltitrierung des Harns besonders wichtigen Stoffe - die Ammoniumsalze und das Glykokoll - sich einwandsfrei mit Phenolphthalein als Indikator formoltitrieren lassen, sei es, daß die Titrierung bis $\mathrm{zu}$ schwach oder bis zu stark roter Farbe geführt wird, scheint es uns ganz fehlerhaft, der Formoltitrierung im Harne eine Sonderstellung zu geben; man muß in diesem wie in allen anderen Fällen bis zu stark roter Farbe mit Phenolphthalein titrieren.

Die Neutralisation der Lösung. Wie schon eingangs erwähnt, hat die Neutralisation den Zweck, die Lösung in den Stand $\mathrm{zu}$ bringen, daß gleich viele Äquivalente von basischen und sauren Gruppen vorhanden sind, so daß z. B. alles Ammoniak als Ammoniumsalz und alles Glykokoll und ähnliche Aminosäuren in freiem Zustande in der Lösung sich befinden.

Es erhebt sich jetzt die Frage: Wie reagieren solche Lösungen von reinem Ammoniumsalz oder von einer reinen Aminosäure den verschiedenen Indikatoren gegenüber? Es soll in dem folgenden nachgewiesen werden, daß solche Lösungen wie die hier erwähnten gegen Lackmus ungefähr neutral, gegen Phenolphthalein dagegen mehr oder weniger stark sauer reagieren, und es soll durch einige Zahlenangaben beleuchtet werden, von welcher Größe der Fehler unter verschiedenen Umständen sein wird, wenn statt Lackmus Phenolphthalein bei der Neutralisation angewendet wird.

a) $20 \mathrm{ccm}$ einer $\mathrm{n} / \mathrm{10}$-Lösung von reinem Ammoniumchlorid zeigten gegen empfindliches Lackmuspapier eine ganz schwache saure Reaktion. Nach Zusatz von 1 Tropfen n/s-Natronlauge war die Reaktion gegen Lackmus schwach alkalisch, aber erst nach Zusatz von im ganzen $0,3 \mathrm{ccm} \mathrm{n} / 5-\mathrm{NaOH}$ nahm die Lösung (mit 0,2 ccm 1/2\% iger Phenolphthaleinlösung versetzt) einen ganz schwach rosa Farbton an. ${ }^{1)}$ Bei weiterem Zutröpfeln von $\mathrm{n} / 5-\mathrm{NaOH}$ nahm die Farbstärke ganz allmählich $z u$, und selbst nach Zusatz von im ganzen $2 \mathrm{ccm} \mathrm{n/s-NaOH}$ war die Lösung kaum so stark rot wie die für die Formoltitrierung dargestellte Kontrollösung (siehe S. 29).

1) Dieser Farbton war so schwach, daß es gewiß unmöglich wäre, denselben in farbigen Flüssigkeiten, z. B. im Harn, zu entdecken. 
$20 \mathrm{ccm}$ der Lösung verbrauchten bei der Formoltitrierung ${ }^{1)} 9,85 \mathrm{ccm}$ $\mathrm{n} / \mathrm{b}-\mathrm{NaOH}$ (berechnet $10 \mathrm{ccm}$ ).

Auf ganz dieselbe Weise wurden eine Reihe anderer Lösungen untersucht.

b) $20 \mathrm{ccm}$ einer $\mathrm{n} / 40$-Lösung von Ammoniumchlorid.

Ganz schwach sauer gegen Lackmus.

Mit 1 Tropfen n/s-NaOH: alkalisch gegen Lackmus.

Mit $0,15 \mathrm{ccm} \mathrm{n} / \mathrm{s}-\mathrm{NaOH}$ : ganz schwach rosa Farbton mit Phenolphthalein.

Mit ca. 0,8 ccm n/s- $\mathrm{NaOH}$ : die Farbstärke der Kontrollösung.

Formoltitrierung: $2,46 \mathrm{ccm} \mathrm{n} / 5-\mathrm{NaOH}$ (ber. $2,5 \mathrm{ccm}$ ).

c) $20 \mathrm{ccm}$ einer $\mathrm{n} / 10-\mathrm{Lösung}$ von reinem Glykokoll.

Ganz schwach sauer gegen Lackmus.

Mit 1 Tropfen $\mathrm{n} / \mathrm{b}-\mathrm{NaOH}$ : neutral gegen Lackmus.

Mit 2 Tropfen $\mathrm{n} / \mathrm{s}-\mathrm{NaOH}$ : ganz schwach alkalisch gegen Lackmus. phthalein.

Mit 0,2 ccm n/s- NaOH: ganz schwach rosa Farbton mit Phenol-

Mit ca. $0,9 \mathrm{ccm} \mathrm{n/5-NaOH}$ : die Farbstärke der Kontrolløsung.

Formoltitrierung: $9,88 \mathrm{ccm} \mathrm{n} / \mathrm{s}-\mathrm{NaOH}$ (ber. $10,0 \mathrm{ccm}$ ).

d) $20 \mathrm{ccm}$ einer n/10-Lösung von Glykokoll.

Kaum wahrnehmbare saure Reaktion gegen Lackmus.

Mit 1 Tropfen $\mathrm{n} / 5-\mathrm{NaOH}$ : deutlich alkalisch gegen Lackmus und eben wahrnehmbarer rosa Farbton mit Phenolphthalein.

Mit 0,3 ccm n/s-NaOH: die Farbstärke der Kontrollösung.

Formoltitrierung: $2,46 \mathrm{~cm} \mathrm{n} / \mathrm{s}-\mathrm{NaOH}$ (ber. $2,5 \mathrm{ccm}$ ).

e) $20 \mathrm{ccm}$ einer $\mathrm{n} / 10-\mathrm{Lösung}$ von reinem Alanin.

f) $20 \mathrm{ccm}$ einer $\mathrm{n} / 40-\mathrm{Lösung}$ von Alanin.

Diese zwei Lösungen verhielten sich im wesentlichen wie die entsprechenden Glykokollösungen.

g) $20 \mathrm{ccm}$ einer $\mathrm{n} / \mathbf{2 0}$-Lösung von Glycylglycin.

Schwach, aber deutlich sauer gegen Lackmus.

Mit 5 Tropfen $\mathrm{n} / \mathrm{s}-\mathrm{NaOH}$ : neutral gegen Lackmus.

Mit 2,25 ccm n/s-NaOH: ganz schwach rosa Farbton mit Phenolphthalein.

Mit ca. $4,0 \mathrm{ccm} \mathrm{n/5-NaOH:} \mathrm{die} \mathrm{Farbstärke} \mathrm{der} \mathrm{Kontrollösung.}$

Formoltitrierung $4,90 \mathrm{ccm} \mathrm{n} / \mathrm{s}-\mathrm{NaOH}$ (ber. $5,0 \mathrm{ccm}$ ).

Während die formolfreie Lösung durch Zusatz von Natronlauge nur ganz allmählich eine stärker und stärker rote Farbe annahm, verlief die Formoltitrierung glatt und scharf; die formolhaltige, ganz schwach rote Lösung wurde mit 2 Tropfen $\mathrm{n} / 5-\mathrm{NaOH}$ noch stärker rot gefärbt als die Kontrollösung.

1) Wenn nicht anders angegeben ist, verstehen wir unter Formoltitrierung Titrierung bis zu stark roter Farbe mit Phenolphthalein als Indikator. 
h) $20 \mathrm{ccm}$ einer $\mathrm{n} / 80$-Lösung von Glycylglycin.

Schwach sauer gegen Lackmus.

Mit 1 Tropfen $\mathrm{n} / \mathrm{s}-\mathrm{NaOH}$ : neutral gegen Lackmus.

Mit 3 Tropfen $n / 5-\mathrm{NaOH}$ : ganz schwach alkalisch gegen Lackmus. phthalein.

Mit 0,55 ccm n/5-NaOH: ganz schwach rosa Farbton mit Phenol-

Mit ca. 1,0 ccm n/n- $\mathrm{NaOH}$ : die Farbstärke der Kontrollösung.

Formoltitrierung: $1,16 \mathrm{ccm} \mathrm{n} / \mathrm{b}-\mathrm{NaOH}$ (ber. $1,25 \mathrm{ccm}$ ).

Aus diesen Versuchen geht hervor, erstens, daß alle untersuchten Lösungen gegen Lackmus schwach sauer reagierten, und daß man daher - streng theoretisch genommen - bei der Neutralisation einen Indikator benutzen sollte, dessen Umschlagspunkt noch saurer als der des Lackmus liegt. Da indessen empfindliches Lackmuspapier sich sonst für diesen Zweck gut geeignet erwiesen hat - der Umschlag z. B. ist scharf und leicht erkennbar - und da, wie es aus den oben angeführten Versuchen zu ersehen ist, der Fehler bei Anwendung von Lackmus gewöhnlich nur ganz geringfügig ist, glauben wir das Lackmuspapier als Indikator bei der Neutralisation der Lösung empfehlen zu können. Es ist leicht verständlich, daß die hier erwähnte Fehlerquelle sich dadurch zu erkennen gibt, daß die nachfolgende Formoltitrierung ein wenig zu niedrige Resultate liefert.

Zweitens ersieht man aus den Versuchsergebnissen, daß man immer einen merkbaren Fehler begeht, wenn man Phenolphthalein als Indikator bei der Neutralisation verwendet, und dieser Fehler, der sich durch viel $\mathrm{zu}$ niedrige Resultate bei der nachfolgenden Formoltitrierung kundbar macht, wird je größer werden, je stärker rot die Farbe, bis zu welcher man titriert, gewählt wird. Besonders groß wird der Fehler sein, wenn derartige Verbindungen wie Glycylglycin vorhanden sind.

Wenn es sich um reine Lösungen von Ammoniumund Aminoverbindungen handelt, kann es somit keinem Zweifel unterliegen, daß Lackmus bei der Neutralisation, dagegen bei der eigentlichen Formoltitrierung Phenolphthalein (bis zu stark roter Farbe) zu verwenden ist.

Sobald aber die in Rede stehende Lösung andere Stoffe 
als die eben erwähnten und zwar besonders, wenn sie schwache Säuren enthält, stellt sich die Sache etwas anders. Die normalen Salze schwacher Säuren sind ja in wässeriger Lösung mehr oder weniger stark hydrolysiert und reagieren daher sowohl gegen Lackmus wie gewöhnlich auch gegen Phenolphthalein mehr oder weniger stark alkalisch. Wenn daher eine Lösung schwacher Säuren gegen Lackmus neutral gemacht worden ist, wird eine kleinere oder größere Alkalimenge notwendig sein, um neutrale oder alkalische Reaktion gegen Phenolphthalein hervorzurufen, und es ist leicht verständlich, daß die im vorhergehenden beschriebene Methode - Neutralisation gegen Lackmus und Formoltitrierung bis zu stark roter Farbe mit Phenolphthalein - in solchem Falle einen kleineren oder größeren Fehler in sich birgt.

Der Harn enthält solche schwachen Säuren, und zwar die für unseren Fall besonders wichtigen Säuren, die Phosphorsäure und die Kohlensäure. Man würde daher einen merkbaren Fehler begehen, wenn man den Harn ohne Vorbehandlung direkt gegen Lackmus neutralisieren und danach mit Phenolphthalein formoltitrieren wollte; denn lackmusneutrale Lösungen von Phosphaten oder Carbonaten erfordern beträchtliche Mengen Alkali, um Phenolphthalein zu röten. ${ }^{1}$ ) Es ist deshalb notwendig, den Harn einer solchen Vorbehandlung zu unterziehen, daß Phosphate und Carbonate vor der Neutralisation und der nachfolgenden Formoltitrierung entfernt werden. Diese Vorbehandlung wird am zweckmäßigsten mit Baryumchlorid und Baryumhydroxyd auf die in der oben erwähnten Abhandlung von V. Henriques (l. c. S. 2) angegebene Weise ausgeführt. Von andern schwachen Säuren, welche in der hier erwähnten Beziehung einen Einfluß haben können, enthält normaler Harn nur ganz minimale Mengen. ${ }^{2}$ ) Der hierdurch

1) Es würde zu weit führen, hier genauere diesbezügliche Daten anzuführen; wir müssen uns damit begnügen, auf die Arbeit von S. P. L. Sörensen: «Enzymstudien II, Über die Messung und die Bedeutung der Wasserstoffionenkonzentration bei enzymatischen Prozessen zu verweisen (Biochemische Zeitschrift, Bd. XXI, S. 131 [1909], und zwar besonders S. 175, 187 und 188).

2) Die Harnsäure spielt, wie wir experimentell nachweisen konnten, Hoppe-Seyler's Zeitschrift f. physiol. Chemie. LXIII. 
bewirkte Fehler muß selbstverständlich äußerst gering sein und hat lediglich zur Folge, daß die bei der Formoltitrierung gewonnenen Resultate ein wenig zu hoch ausfallen. Da somit dieser Fehler den oben erwähnten (s. S. 29 und S. 32) kleinen methodischen Fehlern bei der Formoltitrierung entgegenwirkt, ersieht man, daß die von uns zur quantitativen Bestimmung der Aminosäuren im Harne ausgearbeitete $\mathrm{Me}$ thode wenigstens bei allen normalen Harnen vorzügliche Dienste leistet. Daß z. B. $\beta$-Oxybuttersäure oder andere schwache Säuren, die in pathologischem Harn vorkommen können, zu merkbaren Fehlern Anlaß geben können, ist höchst wahrscheinlich; die Bedeutung derartiger Fehlerquellen $\mathrm{muß}$ aber in jedem speziellen Fall experimentell ermittelt werden.

Schließlich möchten wir noch auf einen Punkt die Aufmerksamkeit lenken. Es ist aus theoretischen Gründen leicht verständlich und es geht außerdem aus den oben beschriebenen Versuchen deutlich hervor, daß durch Zusatz von Lauge (ohne Formol) die Hydroxylionenkonzentration der Lösungen von Ammoniumsalzen oder Aminosäuren nur ganz allmählich ansteigt. Zwischen der Lackmus- und der Phenolphthaleinneutralität ist ein titrimetrisch deutlich merkbarer Abstand vorhanden, und ein noch größerer Abstand enthüllt sich, wenn man von schwach rosa Farbe bis zu stark roter Farbe mit Phenolphthalein als Indikator titriert. Werden dagegen die gleichen Lösungen nach Formolzusatz titriert, so ist der eben erwähnte Abstand zwischen der Titrierung bis zu schwach rosa und bis zu stark roter Farbe wenngleich gewöhnlich nicht ganz verschwunden (vgl. S. 29), doch jedenfalls stark vermindert worden (s. z. B. das Verhalten des Glycylglycins S. 31). Der vorbehandelte (d. h. phosphat- und carbonatfreie) Harn verhält

in dieser Beziehung keine Rolle. $50 \mathrm{ccm}$ einer 0,01-n-Lösung von reinem Mononatriumurat reagierten gegen Phenolphthalein ganz schwach alkalisch und gegen Lackmus deutlich alkalisch, aber schon ein Zusatz von $0,1 \mathrm{ccm}$ n/10-Salzsäure rief neutrale Reaktion gegen Lackmus hervor. Hierzu kommt überdies, wie wir auch gefunden haben, daß so gut wie alle Harnsäure durch die Behandlung des Harns mit Baryumchlorid und Baryumhydroxyd ausgefällt wird. 
sich auf ganz dieselbe Weise, und eben daraus ersieht man, daß es sich um Aminosäuren und ähnliche Verbindungen und nicht um aminofreie schwache Säuren handelt; denn diese letzteren werden durch Formol gar nicht beeinflußt, soda $B$ in diesem Fall die Titrierungen bis zu schwach und bis zu stark roter Farbe - sei es mit, sei es ohne Formol - dieselbe Differenz aufweisen müßten.

Zur weiteren Beleuchtung der Frage werden wir ein einziges Beispiel anführen.

Zur Untersuchung lag ein Harn eines erwachsenen gesunden Mannes vor. $100 \mathrm{ccm}$ des Harnes enthielten nach Henriques-Sörensen $70 \mathrm{mg}$ Ammoniakstickstoff und $33 \mathrm{mg}$ Aminosäurestickstoff. Der vorbehandelte und darauf mit Lackmus als Indikator neutralisierte Harn wurde teils ohne, teils mit Formol und mit Phenolphthalein als Indikator titriert, indem eine passend gefärbte Kontrollösung als Vergleichsflüssigkeit benutzt wurde.

$50 \mathrm{ccm}$ des vorbehandelten, lackmusneutralen Harns $(20 \mathrm{ccm}$ des reinen Harns entsprechend) verbrauchten ohne Formolzusatz $0,75 \mathrm{ccm}$ $\mathrm{n} / \mathrm{s}-\mathrm{NaOH}$, um den schwachen, aber doch deutlichen roten Farbton der Kontrollösung zu erreichen. Es wurden jetzt der Kontrollösung 3 Tropfen $\mathrm{n} / \mathrm{s}-\mathrm{NaOH}$ zugesetzt, wodurch dieselbe eine stark rote Farbe annahm; die Harnlösung aber forderte, um dieselbe Farbstärke zu erreichen, noch $1,2 \mathrm{ccm} \mathrm{n} / \mathrm{s}-\mathrm{NaOH}$. Die öfters erwähnte Differenz betrug also in diesem Fall, indem die 3 Tropfen gleich $0,15 \mathrm{ccm}$ gesetzt werden:

$$
1,2 \div 0,15=1,05 \mathrm{ccm} \mathrm{n} / \mathrm{s}-\mathrm{NaOH} \text {. }
$$

$50 \mathrm{ccm}$ des vorbehandelten lackmusneutralen Harns wurden auf ganz dieselbe Weise, aber mit Formolzusatz titriert. Bei der Titrierung bis zu dem schwachen, aber deutlichen roten Farbton der Kontrolllösung wurden $7,16 \mathrm{ccm} \mathrm{n} / \mathrm{s}-\mathrm{NaOH}$ verbraucht, bei weiterer Titrierung bis zu der stark roten Farbe wurden noch weitere $0,35 \mathrm{ccm} \mathrm{n/s-NaOH}$ verbraucht. Die Differenz betrug folglich in diesem Fall:

$$
0,35 \div 0,15=0,2 \mathrm{ccm} \mathrm{n} / \mathrm{s}-\mathrm{NaOH} \text {. }
$$

In einer früher erschienenen Abhandlung ${ }^{1}$ ) gaben wir eine Methode zur quantitativen Bestimmung des Aminosäurestickstoffs im Harne an. In dieser Abhandlung heißt es Seite 5: «Ob sich im Harn Polypeptide vorfinden, ist noch nicht mit Sicherheit festgestellt worden. Finden sich solche Verbindungen,

1) V. Henriques, Über quantitative Bestimmung der Aminosäuren im Harn. Diese Zeitschrift Bd. LX. 
so ist die Zahl für die Menge des Aminosäurestickstoffs natürlich gar zu niedrig, und zwar um so niedriger, je mehr Aminosäuren aneinander gebunden vorkommen. Übrigens kann es kaum große Schwierigkeit bereiten, mittels der Formoltitrierung vor und nach dem Sieden mit starker Säure zu entscheiden, ob sich solche Polypeptide im Harn finden.»

Um dieses Verhalten näher zu untersuchen, bedienten wir uns folgenden Verfahrens: In $50 \mathrm{ccm}$ Harn bestimmt man in früher beschriebener Weise die Menge des Aminostickstoffs. $\mathrm{Zu}$ anderen $50 \mathrm{ccm}$ Harn setzt man Salzsäure hinzu, und man schüttelt den Harn 6 mal mit Äthylacetat aus, um möglicherweise vorhandene Hippursäure $\mathrm{zu}$ entfernen. Darauf bringt man den Harn in einen Kolben, der einen Zusatz von $50 \mathrm{ccm}$ konzentrierter $\mathrm{HCl}$ erhält, und siedet die Flüssigkeit $11 / 2$ Stunden lang. Nach dem Sieden entfernt man möglichst viel der Salzsäure auf dem Wasserbade. Den Rest löst man in Wasser auf und nach Entfärbung ${ }^{1}$ ) unternimmt man eine Formoltitrierung + einer Ammoniakbestimmung. Finden sich nun Polypeptide im Harn, so muß dieses sich dadurch erweisen, daß die Menge des Aminosäurestickstoffs in dem mit Salzsäure gekochten Harne größer ist als in dem ursprünglichen Harn.

Aus sämtlichen vorliegenden Bestimmungen geht nun hervor, daß man durch Kochen mit Salzsäure eine Abspaltung der Aminosäure erzielt, indem die Formoltitrierung stets eine höhere Zahl in dem mit Salzsäure behandelten als in dem ursprünglichen Harn ergibt. Die Menge des mittels dieses Verfahrens nachweisbaren peptidgebundenen Stickstoffes ist sehr schwankend; die geringste von uns angetroffene Menge betrug 8,9\% (als Prozente der gesamten Menge Aminosäurestickstoffs berechnet; siehe die Tabelle 9./IX. Harn des Menschen); die größte Menge kommt im Hammelurin vor, indem man hier bis $77,7 \%$ peptidgebundenen Stickstoff findet (die Tabelle 10./VII. 09).

Wir bestimmten nicht nur den Aminosäurestickstoff und den Ammoniakstickstoff des Harns, sondern suchten bei der-

1) S. P. L. Sörensen und H. Jessen-Hansen, Über die Ausführung der Formoltitrierung in stark farbigen Flüssigkeiten. Biochem. Zeitschrift, Bd. VII. 
selben Gelegenheit auch die Menge des Hippursäurestickstoffs zu bestimmen. Destilliert man den zum Ausschütteln des Harns benutzten Essigäther ab und kocht man darauf den Rückstand mit konzentrierter Salzsäure, so wird die gesamte Hippursäure sich in Benzoesäure und Glycin spalten, und die Stickstoffmenge des letzteren läßt sich dann nach Neutralisation durch Formoltitrierung bestimmen. Daß man wirklich imstande ist, in dieser Weise den Hippursäurestickstoff $z u$ bestimmen, geht aus folgendem hervor:

In einer Lösung reiner Hippursäure (ca. 3\%) bestimmt man nach Kjeldahls Methode den Stickstoffgehalt als $=23,5 \mathrm{mg}$ in $10 \mathrm{ccm}$. Durch Formoltitrierung nach $11 / 2$ stündigem Kochen mit konzentrierter Salzsäure fanden wir in $12 \frac{1}{2} \mathrm{ccm}$ einen Stickstoffgehalt von 29,12 mg. Es fanden sich also:

Nach Kjeldahl in $100 \mathrm{ccm} 235 \mathrm{mg} \mathrm{N}$

Bei Formoltitrierung » » 233 » »

Ein anderes Beispiel ist folgendes:

$50 \mathrm{ccm}$ Harn (Mensch) werden mit Äthylacetat ausgeschüttelt. Der Rückstand ergab nach Kochen mit $\mathrm{HCl}$ durch Formoltitrierung $2,6 \mathrm{mg} \mathrm{N}$.

Andere $50 \mathrm{ccm}$ Harn erhielten einen Zusatz von $5 \mathrm{ccm}$ Hippursäurelösung mit einem Stickstoffgehalt (Kjeldahl) von 19,1 mg. Der Harn wurde mit Äthylacetat ausgeschüttelt, und der mit $\mathrm{HCl}$ gekochte Rückstand ergab bei Formoltitrierung: $21,9 \mathrm{mg}$ N. Die zugesetzte Menge Hippursäurestickstoff (19,1 mg) + dem im Harn befindlichen Hippursäurestickstoff $(2,6 \mathrm{mg})$ gibt $21,7 \mathrm{mg} \mathrm{N}$. Berücksichtigt man, daß die Bestimmung an einer Lösung unternommen wurde, die $16 \mathrm{ccm}$ Harn entspricht, so ist die Übereinstimmung eine außergewöhnlich gute $\mathrm{zu}$ nennen. Hat man hinlänglichen Harn zur Verfügung, so kann man mittels dieser leicht ausführbaren und schnellen Methode sehr zuverlässige Bestimmungen des Hippursäurestickstoffs im Harn erzielen.

Um die oben angeführten Bestimmungen zu kontrollieren, bestimmten wir in der Regel gleichzeitig die Menge des gesamten formoltitrierbaren Stickstoffes in $50 \mathrm{ccm}$ Harn nach Sieden mit Salzsäure - ohne vorhergehendes Ausschütteln mit 
Äthylacetat. Benutzen wir der Übersichtlichkeit wegen folgende Bezeichnungen (siehe die untenstehende Tabelle):

$A=$ Aminosäurestickstoff im ursprünglichen Harn,

$\mathrm{B}=$ Aminosäurestickstoff im Harn nach Ausschütteln mit Äthylacetat und darauf folgendem Kochen mit $\mathrm{HCl}$,

$\mathrm{C}=$ Aminosäurestickstoff im Harn nach Kochen mit $\mathrm{HCl}$,

$\mathrm{D}=$ Aminosäurestickstoff in der im Äthylacetate gelösten Hippursäure nach Kochen mit $\mathrm{HCl}$, so folgt hieraus, daß wir stets $\mathrm{B}+\mathrm{D}=\mathrm{C}$ haben müssen.

Betrachtet man die in der Tabelle angeführten Zahlen, so wird man finden, daß die Resultate ganz gut miteinander übereinstimmen; in einigen Fällen ist die Übereinstimmung ganz vorzüglich, in anderen dagegen weniger gut. $\mathrm{Zu}$ beachten ist hier jedoch, daß die im Hunde- und Menschenharn angetroffenen Mengen Hippursäure, wenn man höchstens $50 \mathrm{ccm}$ Harn anwendet, so gering sind, daß man keine völlige Übereinstimmung verlangen darf.

$\mathrm{Zu}$ erwähnen ist noch, daß man bei Bestimmungen im Harne von ausschließlich mit Fleisch (500-1000 g) ernährten Hunden nicht imstande ist, übereinstimmende Resultate zu erzielen. Der Grund hierfür ist wahrscheinlich die große Menge Ammoniak, die sich während des Kochens des Harns mit Salzsäure bildet. So fand man - um ein Beispiel zu nennen im ursprünglichen Harn $22 \mathrm{mg}$ Ammoniak- + Aminosäurestickstoff, während nach Kochen mit Salzsäure die Menge bis auf $287 \mathrm{mg} \mathrm{N}$ stieg. Diese große Menge Ammoniak macht natürlich die Bestimmung des Ammoniakstickstoffes und mithin auch die Bestimmung des Aminosäurestickstoffes unsicher. $\mathrm{Ob}$ dies die einzige Ursache der weniger guten Resultate mit Bezug auf den Fleischharn ist, muß vorläufig dahingestellt bleiben. Selbstverständlich versuchten wir es, vor der Formoltitrierung das Ammoniak zu entfernen, u. a. durch Destillation im Vakuum; keine der angewandten Methoden führte indes zu einem völlig befriedigenden Resultate. In der Tabelle sind nur einzelne Beispiele von Analysen des Hundeharns nach Fleischfütterung angegeben; wie man sieht, stimmen die Zahlen nur so einigermaßen miteinander überein. 


\begin{tabular}{|c|c|c|c|c|c|c|c|c|}
\hline & \multirow{2}{*}{$\begin{array}{l}\text { Diu- } \\
\text { rese }\end{array}$} & \multirow{2}{*}{$\begin{array}{c}\text { Total- } \\
\mathrm{N} \\
\mathrm{g}\end{array}$} & \multicolumn{2}{|c|}{$\begin{array}{c}\text { Aminosäure- } \\
\mathrm{N}\end{array}$} & \multirow{2}{*}{$\begin{array}{c}\text { Amino- } \\
\text { säure- } \\
+ \\
\text { Hippur- } \\
\text { säure-N } \\
\text { C. } \\
\text { g }\end{array}$} & \multirow{2}{*}{$\begin{array}{c}\text { Hippur- } \\
\text { säure- } \\
\text { N } \\
\text { D. } \\
\text { g }\end{array}$} & \multirow{2}{*}{$\begin{array}{c}\text { Am- } \\
\text { mo- } \\
\text { niak- } \\
\mathrm{N} \\
\mathrm{g}\end{array}$} & \\
\hline & & & $\begin{array}{l}\text { A. } \\
\text { g }\end{array}$ & $\begin{array}{c}\text { B. } \\
\text { g }\end{array}$ & & & & \\
\hline 10./VII. 09 & 960 & 14,592 & 0,064 & 0,287 & 0,817 & 0,542 & 0,306 & \\
\hline 12. & 960 & 12,960 & 0,082 & 0,225 & - & 0,538 & 0,204 & Hammel- \\
\hline 13. & 900 & 12,060 & 0,073 & 0,236 & 0,730 & 0,496 & 0,360 & urin. \\
\hline 14. & 1000 & 13,350 & 0,100 & 0,275 & - & 0,560 & 0,306 & Heufutter. \\
\hline 15. & 780 & 10,530 & 0,086 & 0,180 & 0,641 & $0,50 \check{~}$ & 0,344 & \\
\hline 9./IX. 09 & 1130 & 8,814 & 0,174 & 0,191 & - & 0,040 & 0,393 & \\
\hline 10. & 850 & 11,390 & 0,190 & 0,265 & 0,295 & 0,037 & 0,568 & \\
\hline 11. & 1550 & 11,315 & 0,189 & - & 0,302 & 0,041 & 0,557 & \\
\hline 13. & 1250 & 13,688 & 0,230 & 0,302 & 0,354 & 0,066 & 0,699 & \\
\hline 27./V. 09 & 320 & 15,360 & 0,113 & 0,137 & 0,135 & 0,011 & 0,430 & \\
\hline 2./VII. & 300 & 15,090 & $0,15 k$ & 0,229 & 0,218 & 0,011 & 0,408 & \\
\hline 3. & 260 & 14,534 & 0,118 & 0,171 & 0,188 & 0,007 & 0,341 & \\
\hline 11./V. & 130 & 3,400 & 0,035 & 0,044 & 0,051 & 0,007 & 0,242 & \\
\hline 13. & 160 & 4,064 & 0,052 & 0,071 & 0,073 & 0,003 & 0,317 & \\
\hline 15. & 300 & 4,530 & 0,057 & 0,075 & 0,086 & 0,011 & 0,470 & \\
\hline 18. & 165 & 4,158 & 0,058 & 0,071 & 0,075 & 0,008 & 0,279 & \\
\hline 22. & 200 & 5,160 & 0,059 & 0,079 & 0,085 & 0,007 & 0,449 & \\
\hline 18./VI. & 175 & 5,268 & 0,044 & 0,058 & 0,062 & 0,006 & 0,391 & Hund. \\
\hline 19. & 620 & 5,766 & 0,059 & 0,078 & 0,082 & 0,011 & 0,471 & Brot. \\
\hline 23. & 140 & 3,465 & 0,042 & 0,064 & 0,068 & 0,004 & 0,232 & \\
\hline 25. & 300 & 5,970 & 0,087 & 0,148 & 0,158 & 0,007 & 0,435 & \\
\hline 26. & 200 & 4,750 & 0,064 & 0,074 & 0,088 & 0,007 & 0,365 & \\
\hline 28. & 280 & 4,676 & 0,066 & 0,078 & 0,090 & 0,005 & 0,269 & \\
\hline 29. & 330 & 5,033 & 0,085 & 0,104 & 0,105 & 0,003 & 0,368 & \\
\hline
\end{tabular}


40 V. Henriques und S. P. L. Sörensen, Über Aminosäuren usw.

Aus den in der Tabelle angeführten Zahlen geht übrigens hervor, daß die Menge des peptidgebundenen Stickstoffes (in Prozenten des gesamten vorhandenen Aminosäurestickstoffes ausgedrückt) stark variiert. Im Hammelurin findet man zwischen 52,2 und 77,7\% (durchschnittlich 65,2) peptidgebundenen Stickstoffs, während die Menge im menschlichen Harn zwischen 8,9 und 28,3\% schwankt (im Durchschnitt $=22,2 \%$ ). Im Hundeharn fanden sich bei Fleischfütterung zwischen 17,5 und $32,8 \%$ (durchschnittlich 27,1), bei Brotfütterung von 13,5 bis $41,2 \%$ (durchschnittlich 23,8) peptidgebundenen Stickstoffes. 